Apidologie, 1984, 15 (2), 205-210

\title{
SPEZIFITÄT EINES BRUTPHEROMONS UND BRUTERKENNUNG BEI DER HONIGBIENE (APIS MELLIFERA L.)
}

\author{
N. KOENIGER* und H.J. VEITH** \\ *Institut für Bienenkunde (Polytechnische Gesellschaft), \\ Fachbereich Biologie der Universität Frankfurt a. M., \\ Karl-von-Frisch-Weg 2, D - 637 Oberursel \\ **Institut fïr Organische Chemie und Biochemie der Technischen Hochschule \\ D - 6100 Darmstadt
}

\section{ZUSAMMENFASSUNG}

Das Brutpheromon der Honigbiene, Gyceryl-1,2dioleat-3-palmitat, löste im Verhaltenstest die Belagcrung von Königinnenzellattrappen aus. Andere Triglyceride, Triolein und Tripalmitin, sowie das zum Brutpheromon Stellungsisomere 1,3-diolein-2-palmitin zeigten im Test keine Wirkung. Zwischen einem racemischen Gemisch des Pheromons sowie den reinen optischen Isomeren ergab sich kein Unterschied. Alle diese Substanzen lösten Pflegeverhalten aus.

Arbeiterinnenpuppen enthielten jeweils 2-5 $\mu \mathrm{g}$ Pheromon, Drohnenpuppen $10 \mu \mathrm{g}$ und Königinnenpuppen je $30 \mu \mathrm{g}$. Imagines (Arbeiterinnen und Drohnen) hatten kein Glyceryl-1,2-dioleat-3-palmitat. Diese Befunde wurden im Zusammenhang mit einer allgemeinen Hypothese der Bruterkennung bei der Honigbiene diskutiert.

Die Aufzucht der Brut bei der Honigbiene erfordert eine zeitliche Folge von einzelnen sehr verschiedenen Pflegemaßnahmen. Nur in genauer Anpassung an den jeweiligen Zustand des Eis, der Made oder der Puppe kann das « richtige » Verhalten von den Pflegebienen praktiziert werden. Welche Signale der Brut für diese Kommunikation benutzt werden, ist weitgehend unbekannt.

Durch die kürzlich gelungene Identifizierung eines Puppenpheromons (KoENIGER und Veith, 1983), besteht jetzt die Möglichkeit experimentelle Hinweise auf die Spezifität dieses Signals zu erhalten. Darüber hinaus können Befunde über das Vorkommen des Pheromons bei Imagines und verschiedenen Brutstadien Hinweise liefern, ob die Hypothese über eine allgemeine Bruterkennung wahrscheinlicher ist als die Annahme, daß jedes Brutpflegeverhalten von spezifischen und voneinander unabhängigen, verschiedenen Reizen ausgelöst wird. 


\section{MATERIAL UND METHODE}

Die Versuche wurden mit Bienen der Rasse Apis mellifera carnica durchgeführt. Es wurden Linienkombinationen $07 \times 1075$ verwendet, die sich bei ähnlichen Versuchen (KoENIGER, 1978) bereits bewährt haben und die sich durch große "Wabenstetigkeit » auszeichnen.

Der Biotest wurde mit Stockbienen durchgeführt. Dazu wurden Waben, die verdeckelte Brut enthielten, aus der Beute entnommen und für $15 \mathrm{~min}$. einzeln und frei bei Tageslicht aufgestellt. In dieser Zeit flog ein Teil (ca. 30\%) der aufsitzenden Bienen ab. Der verbliebene Rest wurde dann in einen «Marburger Feglingskasten» abgekehrt. Auch bei diesem Vorgang flog ein weiterer Teil der Bienen ab. Der Kasten wurde dann verschlossen und von 10-18 Uhr in einem dunklen Raum bei $14^{\circ} \mathrm{C}$ aufbewahrt.

Gegen 18 Uhr wurden die Bienen in die Testkästen (Abb. 1) gefüllt und ihr Verhalten stündlich protokolliert. In der Regel hatten sich die Bienen nach 2-3 $\mathrm{h}$ entschieden und belagerten nur eine der beiden gebotenen Zellen.

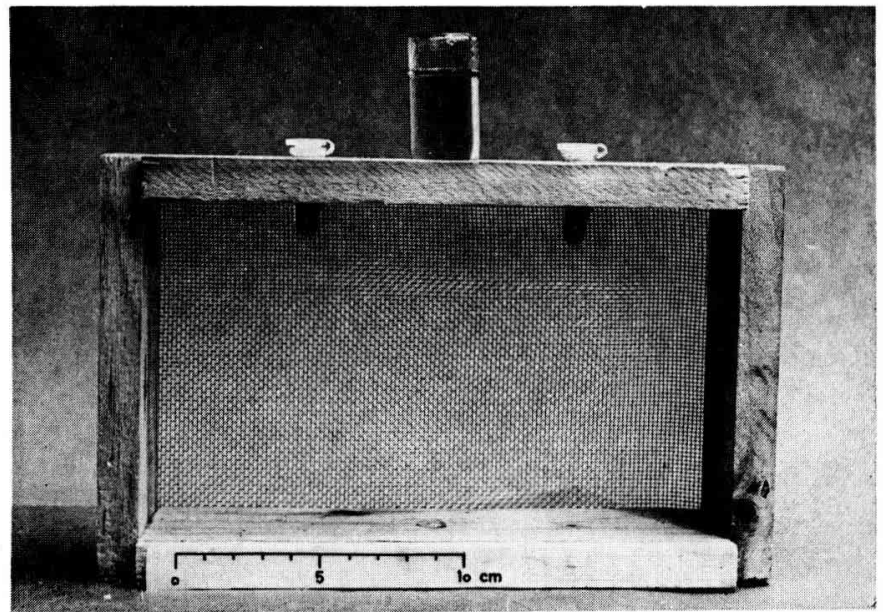

ABB. 1. - Versuchskasten für Biotest

In der Decke des Kastens befinden sich die semiartifiziellen Königinnenzellen und in der Mitte ein Futtergefäß gefüllt mit Honig.

Fig. 1. - Testbox for bioassay

In the top side of the box 2 semiartificial queencells and in the center a boney container are offered.

Die Testsubstanzen wurden in Diethylether gelöst und vor dem Versuch auf den natürlichen, wachsfreien Seidenkokon der semiartifiziellen Königinnenzelle (Abb. 2) aufgetragen. Die Kontrollzelle wurde mit gleicher Menge Lösungsmittel (Ether) imprägniert.

Zur quantitativen Bestimmung des Pheromons wurden Extrakte mit Diethylether verwandt (Extraktionszeit $60 \mathrm{~min}$.). Die Reinigung der Extrakte und die massenspektroskopische Quantifizierung wurde nach der bei Koeniger und VeITH (1983) angegeben Methode durchgeführt. 


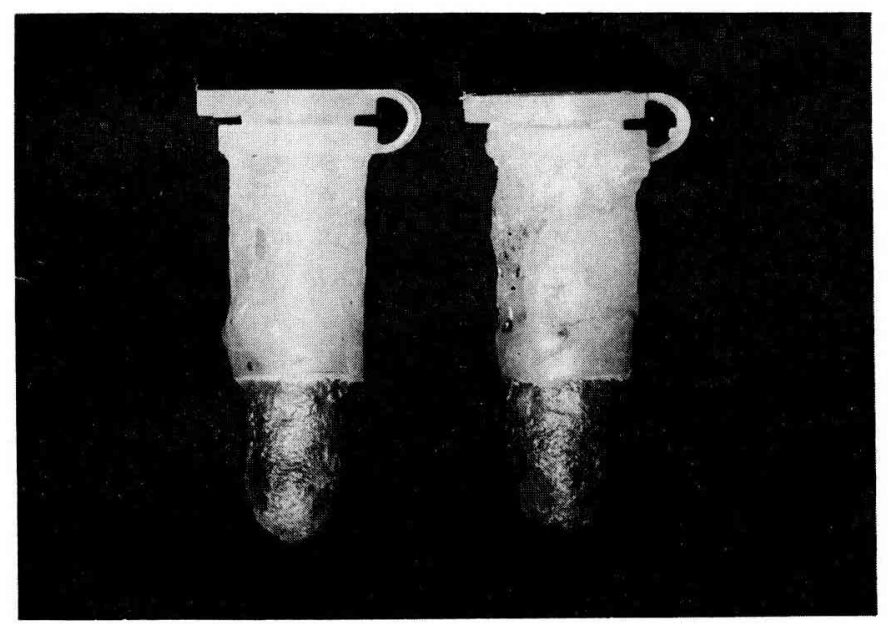

Aвв. 2. - Semiartifizielle Königinnenzellen

Ein natürlicher Puppenkokon wurde durch Extraktion mit Ether vom Wachs gereinigt und an ein Plastikgefäß geklebt.

FIG. 2. - Semiartificial queencells

Natural cocons of queencells were extracted in ether to remove the wax and glued to a small plastic vial.

\section{ERGEBNISSE}

\section{Spezifität des Pheromons}

Es wurden zunächst mit negativem Erfolg die Triglyceride Triolein und Tripalmitin gestestet. Weiter wurde das stellungsisomere Triglycerid 1,3-dioleat-2palmitat geboten. Auch dabei ergab sich kein Unterschied zu den mit reinem Ether imprägnierten Kontrollzellen. Die optischen Isomeren sowie das Racemat des Pheromons waren attraktiv und zeigten keine Unterschiede (Tab. 1).

\section{Quantifizierung des Pheromons}

Für Puppen mit dunklen Augen wurde die Menge des vorhandenen Pheromons bestimmt. Für Königinnenpuppen erhielten wir mit 20-40 $\mu \mathrm{g}$ die höchsten Werte. Drohnenpuppen enthielten ca. $10 \mu \mathrm{g}$. Bei Arbeiterinnen wurden ca. 2-5 $\mu \mathrm{g}$ gefunden. Weiter zeigten Imagines (Arbeiterinnen und Drohnen) bei einer Meßgenauigkeit von 0,2 ftg ein negatives Ergebnis, d.h. wir konnten das 1,2-dioleat-3-palmitat nicht nachweisen.

Bei Maden gelang der Nachweis des Pheromons, jedoch reichen die Messungen noch nicht für eine zuverlässige Quantifizierung aus. 
TAB. 1. - Spezifität des Brutpheromons

TABL. 1. - Specificity of the brood pheromone

\begin{tabular}{l|c|c|c|c}
\hline \hline \multicolumn{1}{c|}{$\begin{array}{c}\text { Substanz } \\
\text { Substance }\end{array}$} & $\begin{array}{c}\text { Dosis / Zelle } \\
\text { Dose / cell }\end{array}$ & $\begin{array}{c}\text { Isomerie } \\
\text { Isomerism }\end{array}$ & $\begin{array}{c}\mathrm{n} \text { (Tests) } \\
\mathrm{n} \text { (tests) }\end{array}$ & $\begin{array}{c}\text { Test : Kontrolle } \\
\text { Test : control }\end{array}$ \\
\cline { 2 - 4 } $\begin{array}{l}\text { Triolein } \\
\text { Tripalmitin }\end{array}$ & $\begin{array}{c}400 \mu \mathrm{g} \\
400 \mu \mathrm{g}\end{array}$ & $\begin{array}{c}\text { Racemat } \\
\text { Racemat }\end{array}$ & 29 & $55 \%: 45 \%$ \\
$\begin{array}{l}\text { Glyceryl-1,3-dioleat- } \\
\text { 2-palmitat }\end{array}$ & $100 \mu \mathrm{g}$ & Racemat & 39 & $51 \%: 49 \%$ \\
$\begin{array}{l}\text { Glyceryl-1,3-dioleat- } \\
\text { 2-palmitat }\end{array}$ & $100 \mu \mathrm{g}$ & Racemat & 21 & $90 \%: 10 \%$ \\
$\begin{array}{l}\text { Glyceryl-1,3-dioleat- } \\
\text { 2-palmitat }\end{array}$ & $100 \mu \mathrm{g}$ & $\mathrm{S}$ & 22 & $77 \%: 23 \%$ \\
$\begin{array}{l}\text { Glyceryl-1,3-dioleat- } \\
\text { 2-palmitat }\end{array}$ & $100 \mu \mathrm{g}$ & $\mathrm{R}$ & 20 & $85 \%: 15 \%$ \\
\hline
\end{tabular}

\section{DISKUSSION}

Die hier getestete Reaktion beruht in erster Linie auf einer Kontaktperzeption. Die Bienen kommen im Testkasten direkt mit den Substanzen, die auf den Kokon der Testzelle aufgetragen werden, in Berührung. Auch bei anderen sozialen Hymenopteren beruht die Bruterkennung oft auf Kontaktchemoperzeption (KoENIGER, 1978).

Das erste identifizierte Brutpheromon bei der Ameise Solenopsis invicta ist gut von anderen Triglyceriden unterschieden. Auch das Stellungsisomere 1,3diolein-2-palmitin zeigt keine Wirksamkeit im Test. Dagegen können die Pflegepalmitin, unterscheidet.

Das hier untersuchte Brutpheromon wird offenbar von den Bienen im Biotest gut von anderen Triglyceriden unterscheiden. Auch das Stellungsisomere 1,3diolein-2- palmitin zeigt keine Wirksamkeit im Test. Dagegen können die Pflegebienen die optischen Isomeren offenbar nicht untercheiden. Das Racemat wie auch R- und S-1,2-diolein-3-palmitin sind gleichermaßen wirksam.

Danach scheint die Perzeption weniger spezifisch zu sein als bei vielen anderen Insektenpheromonen, bei denen häufig optische Isomeren verschieden wirksam sind (JACOBSON, 1972). Dieser Unterschied beruht wahrscheinlich darauf, daß eine Erkennung von optischen Isomeren nur bei olfaktorischen Rezeptoren möglich ist, während für Kontaktchemorezeptoren eine solche Leistung bisher nicht nachgewiesen ist. 
Die Quantifizierung des Brutpheromons bei den Puppen steht in guter Übereinstimmung zu Ergebnissen von Attraktivitätstests (KoENIGER, 1978). Dabei hatten sich Königinnenpuppen, die ca. $30 \mu \mathrm{g}$ Pheromon enthalten, am attraktivsten erwiesen. An zweiter Stelle kamen Drohnenpuppen, die mit ca. $10 \mu \mathrm{g}$ Pheromon etwa fünf Mal attraktiver waren als Arbeiterinnenpuppen, die nur ca. 3,5 $\mu \mathrm{g}$ des Pheromons besitzen. Es ist geplant, diesen Aspekt weiter zu untersuchen. Wenn die Attraktivität von verdeckelter Arbeiterinnenbrut von der Menge des Brutpheromons abhängt, dann könnte sich durch Applikation von zusätzlichem Pheromon ein Attraktivitätsgleichgewicht zu Drohnenbrut herstellen lassen. Königinnenbrutzellen waren wesentlich attraktiver als Arbeiterinnen- oder Drohnenbrut. Hier spielt wahrscheinlich neben den verschiedenen Pheromonmengen auch die prinzipiell andere Zellform eine Rolle.

Bei Imagines findet sich bei einer Meßgenauigkeit von 0,2 $\mu \mathrm{g}$ kein 1,2-dioleat3-palmitat. Das gefundene Pheromon scheint dagegen auch bei Larven vorhanden zu sein. So handelt es sich wohl um eine für Brut spezifische Substanz, deren Funktion bisher jedoch nur im Zusammenhang mit der Attraktivität von Königinnenzellen untersucht ist.

Sollte sich im Rahmen weiterer Versuche ergeben, daß dieses Pheromon auch bei anderen Brutpflegeverhalten eine Rolle spielt, so handelt es sich hier um eine allgemeinere Bruterkennungssubstanz. Das spezifische, an die biologische Situation angepaßte Pflegeverhalten würde nach dieser Hypothese jeweils durch andere Reize bestimmt, die synergistisch zu dem Brutpheromon wirken würden. Für das Wärmen der Königinnenzelle trifft diese Überlegung zu. Erst das gleichzeitige Vorhandensein von Pheromon, mechanischen Auslösern und dem intakten Kokon führt zu einer Thermoregulation (KoENIger, 1978). Jeder dieser Reize ist für sich allein nicht biologisch wirksam.

Eingegangen im April 1984.

\author{
RESUME \\ SPECIFICITE D'UNE PHEROMONE DE COUVAIN \\ ET RECONNAISSANCE DU COUVAIN CHEZ L'ABEILLE (APIS MELLIFICA)
}

La phéromone de couvain glyceryl-1,2-dioleate-3 palmitate, déclenche le regroupement des abeilles sur les cellules royales factices. D'autres triglycérides, trioléine et tripalmitine, ne déclenchent pas ce comportement. L'isomère glyceryl-1,3-dioleate-2-palmitate, est également inefficace. Il n'y a pas de différence entre un mélange racémique de la phéromone et les isomères optiques purs; ces produits déclenchent le comportement de soin. Les nymphes d'ouvrières contiennent chacune de 2 à $5 \mu \mathrm{g}$ de phéromone; celles de mâles, $10 \mu \mathrm{g}$ et celles de reines, $30 \mu \mathrm{g}$. Les adultes (ouvrières et reines) ne renferment pas de glyceryl-1,2-dioleate-3-palmitate. Ces résultats sont discutés dans le cad:e d'une hypothèse générale de la reconnaissance du couvain chez l'abeille. 


\section{SUMMARY \\ SPECIFITY OF A BROOD PHEROMONE AND BROOD RECOGNITION IN THE HONEY BEE (APIS MELLIFERA)}

The brood pheromone, Glyceryl-1,2-dioleate-3-palmitate, elicited the clustering of bees on dummy queen cells. Other triglycerides, triolein and tripalmitin did not release this behaviour. The isomere Glyceryl-1,3-dioleate-2-palmitate was also ineffective, whereas the pure optical isomeres and a racemic mixture of the pheromone released clustering, and no differences among them. were detected in the assay.

Worker pupae contained $2-5 \mu \mathrm{g}$ pheromone; drone pupae, $10 \mu \mathrm{g}$; and queen pupae, $30 \mu \mathrm{g}$ each. Imagines (worker and drones) contained no Glyceryl-1,2-dioleate-3-palmitate. These data are discussed in connection with a general hypothesis on brood recognition in the honey bee.

\section{LITERATUR}

Bigeley W.S., VinSON S.B., 1975. - Characterisation of a brood pheromone isolated from the sexual brood of the imported Fire Ant, Solenopsis invicta. Ann. Entomol. Soc. Am., 68, 301-304.

JACOBson M., 1972. - Insect sex pheromones. Acad. Press, New York, London.

Koeniger N., 1978. - Das Wärmen der Brut bei der Honigbiene (Apis mellifera). Apidologie, 9 (4), 301-304.

Koeniger N., VeITH H.J., 1983. - Identification of a triglyceride (Glyceryl-1,2-dioleate-3-palmitate) as a brood pheromone of the honeybee (Apis mellifera). Apidologie 14 (1), 59. 